\title{
Reply from Mr Kennedy
}

\section{Dear Sir}

Dr Burke raises a number of concerns in his letter about the reliability and validity of the Functional Independence Measure (FIM) and specific issues to do with the statistical analysis and conclusions of our study of clinical and self ratings. Before dealing with some of the specific issues, I would like to address the general issue of the FIM.

Dr Burke describes the FIM as a 'measurement tool which at best is of unproven validity and reliability'. In our paper we reviewed some of the most pertinent reliability and validity studies. It is our opinion that these studies, and other studies that have recently been done on the FIM, indicate that it is an appropriate basic indicator of the severity of disability. This is not only our opinion, but that of both the American Spinal Injuries Association and International Medical Society of Paraplegia who have adopted the FIM as the standard measure of functional independence in persons with spinal cord injury. No measure is without its shortcomings and our study aimed to inform the scientific community of our observation of the similarity between clinical and self ratings of patients on the FIM.

Dr Burke also raises a number of other specific issues. He mentions that there was a need for cross checking between therapists and nurses, and that patients' performance fluctuated between therapy departments and the ward. On page 3 of the 1987 manual, the authors highlight that the FIM is a measure of what the subject actually does, not what he or she ought to be able to do. Also, on the same page, the authors highlight that the FIM is intended to be discipline-free, to be used by any clinician regardless of discipline. There is no suggestion of cross checking between therapists. It is true that the staff involved did not receive formal training or accreditation. However, at the time of the study no formal training existed in the UK but staff were trained as appropriate by the research team using instructions from the manual. Whilst the patients were not formally trained, at the time of the self report, they had completed the FIM with a trained researcher, on at least four occasions. (In the paper we mentioned the average time, post injury, upon discharge was 24.75 weeks; patients were assessed on the FIM every 6 weeks). Furthermorc, as mentioned in our paper, Fuhrer et al (1992) have developed a self-report shortened form of the FIM.

Our study aimed to compare the relative efficacy of using the FIM as a self-report measure versus clinician involvement. Instructions for the administration of the FIM are for support of the Uniform Data Set to document the severity of patient disability and the outcomes of medical rehabilitation in the United States of America. This paper used different times to pursue the specific hypotheses raised.

With respect to the comments by Dr Burke about statistical jargon, invalid conclusions, and the paragraph concerning the number of patients, i.e. 36 out of 40 and 27 out of 40, (and whether one can possibly compare these), in the paper we highlight that 40 patients were studied while in hospital and 40 patients were studied post discharge. These were matched pairs that were used for statistical analysis. I would hardly describe the provision of means, standard deviations and correlation coefficients as 'statistical jargon'. These basic descriptive statistics have highlighted the similarity of the results between the two groups. An 'eyeball' test would confirm this. The statistical procedures implemented were appropriate and did not violate any of the statistical assumptions. I agree that the phrase concerning the compliance rates is misleading, but this does refer to the wider cohort from which this sample was drawn.

We concluded that the self-report Functional Independence Measure can be a useful tool in conjunction with clinical observation. This is a modest study, exploring a modest issue, making modest conclusions. We do believe that research has demonstrated that the FIM has appropriate levels of interrater reliability, and has demonstrated concurrent validity, content validity and face validity. Our study reported our observation of the similarity between self-report and clinical ratings. We thought this would be of interest to people involved with the Functional Independence 
Measure, and whilst it is a small sample, we felt the results from 40 patients would be a useful indicator to other people involved in the investigation of this measure of functional outcome.

If Dr Burke has any further comments, I would be grateful to receive them.

Paul Kennedy

Consultant Clinical Psychologist

National Spinal Injuries Centre

Stoke Mandeville Hospital

Aylesbury, Bucks HP21 8AL, England 